\title{
Whole-exome sequencing identifies multiple pathogenic variants in a large South Indian family with primary open-angle glaucoma
}

\author{
Mohd Hussain Shah ${ }^{1, \#}$, Manojkumar Kumaran ${ }^{2,3, \sharp}$, Prakash Chermakani ${ }^{1,4}$, Mohideen Abdul Kader, \\ R Ramakrishnan ${ }^{5}$, Subbiah R Krishnadas ${ }^{4}$, Bharanidharan Devarajan ${ }^{2, *}$, Periasamy Sundaresan $^{1}$
}

\begin{abstract}
Purpose: To identify the pathogenic variants associated with primary open-angle glaucoma (POAG) using whole-exome sequencing (WES) data of a large South Indian family. Methods: We recruited a large five-generation South Indian family $(n=84)$ with a positive family history of POAG $(n=19)$. All study participants had a comprehensive ocular evaluation. We performed WES for 16 samples (nine POAG and seven unaffected controls) since Sanger sequencing of the POAG candidate genes (MYOC, OPTN, and TBK1) showed no genetic variation. We used an in-house pipeline for prioritizing the pathogenic variants based on their segregation among the POAG individual. Results: We identified one novel and five low-frequency pathogenic variants with consistent co-segregation in all affected individuals. The variant c.G3719A in RPGR-interacting domain of RPGRIP1 that segregated heterozygously with the six POAG cases is distinct from variants causing photoreceptor dystrophies, reported affecting the RPGR protein complex signaling in primary cilia. The cilia in trabecular meshwork (TM) cells has been reported to mediate the intraocular pressure (IOP) sensation. Furthermore, we identified a novel c.A1295G variant in Rho guanine nucleotide exchange factors Gene 40 (ARHGEF40) and a likely pathogenic variant in the RPGR gene, suggesting that they may alter the RhoA activity essential for IOP regulation. Conclusion: Our study supports that low-frequency pathogenic variants in multiple genes and pathways probably affect Primary Open Angle Glaucoma's pathogenesis in the large South Indian family. Furthermore, it requires larger case-controls to perform family-based association tests and to strengthen our analysis.
\end{abstract}

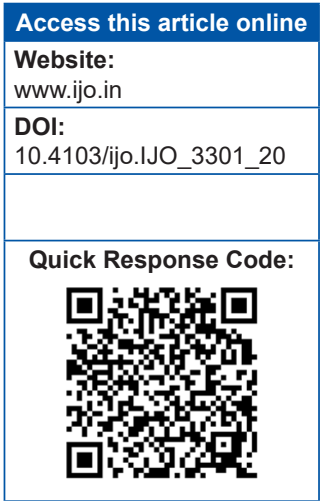

Key words: ARGHEF40 gene, Genetic heterogeneity, primary open-angle glaucoma, RPGRIP1 South Indian, whole-exome sequencing

Glaucoma is typically characterized by progressive degeneration of the optic nerve, which causes irreversible blindness. It is the second leading cause of global blindness after cataract. ${ }^{[1]}$ Primary open-angle glaucoma (POAG) is a subset of glaucoma majorly associated with loss of retinoganglion cells. Their axons trigger permanent vision loss with an apprehensive exponential growth affecting around 60.5 million people worldwide. Due to the exponential increase in the global aging population, it is estimated that 80 million people will be affected by POAG by the end of $2020^{[2]}$ and the count could be expected to rise 111.8 million people by 2040 and thus has an inexplicable impact on Asian and African population. ${ }^{[3]}$ Asia alone accounts for approximately $60 \%$ of global glaucoma, whereas the Africa population represents $(13 \%)$, the second-largest proportion of glaucoma cases globally. In India, it is estimated that 12 million people have been affected by glaucoma. ${ }^{[4,5]}$ This number is expected to increase by 16 million by the end

Departments of ${ }^{1}$ Genetics and ${ }^{2}$ Bioinformatics, Aravind Medical Research Foundation, ${ }^{6}$ Glaucoma Clinic, Aravind Eye Hospital, Madurai, ${ }^{5}$ Glaucoma Clinic, Aravind Eye Hospital, Tirunelveli, ${ }^{3}$ School of Chemical and Biotechnology, SASTRA (Deemed to be University), Thanjavur, ${ }^{4}$ Department of Molecular Biology, Alagappa University, Karaikudi, Tamil Nadu, India

${ }^{*}$ Authors are equally contributed

Correspondence to: Dr. Periasamy Sundaresan, Department of Genetics, Aravind Medical Research Foundation, Madurai, Tamil Nadu, India. E-mail: sundar@aravind.org

Received: 21-Oct-2020

Revision: 21-Feb-2021

Accepted: 05-Apr-2021 of 2020. ${ }^{[4,5]}$ POAG is associated with several external risk factors, including advanced age, central corneal thickness, myopia, steroid responsiveness, and elevated intraocular pressure (IOP). ${ }^{[6]}$ However, these risk factors do not capture the full spectrum of the disease. Though, positive family history is also one of the risk factors for POAG. Genetic characterization of the POAG positive family history is useful for identifying POAG-candidate genes (MYOC, OPTN, and TBK1) $)^{[6-8]}$ that are capable of causing POAG. However, these candidate genes were discovered through large pedigrees with a positive family history of glaucoma. In addition, many studies have shown that POAG development is associated with various genetic risk factors, including genetic variants in CDKN2B-AS ${ }^{[9-12]}$ CAV1/CAV2, ${ }^{[13]}$ TMCO1 $^{[14]}$ AFAP1, ${ }^{[15]}$ TXNRD2, FOXC1/GMDS, ATXN2, ${ }^{[16]}$ FNDC3B, ${ }^{[17,18]}$ GAS, ${ }^{[14]}$ PMM2, ${ }^{[19]}$ TGFBR3, ${ }^{[20]}$ and SIX1/SIX6. ${ }^{[10,11]}$ The genetic heterogeneity and definitive heritability of the disease necessitated an extensive molecular characterization to identify the factors responsible for the genetic predisposition of POAG in affected individuals.

This is an open access journal, and articles are distributed under the terms of the Creative Commons Attribution-NonCommercial-ShareAlike 4.0 License, which allows others to remix, tweak, and build upon the work non-commercially, as long as appropriate credit is given and the new creations are licensed under the identical terms.

For reprints contact: WKHLRPMedknow_reprints@wolterskluwer.com

Cite this article as: Shah $\mathrm{MH}$, Kumaran $\mathrm{M}$, Chermakani $\mathrm{P}$, Kader MA, Ramakrishnan R, Krishnadas SR, et al. Whole-exome sequencing identifies multiple pathogenic variants in a large South Indian family with primary open-angle glaucoma. Indian J Ophthalmol 2021;69:2461-8. 
Our previous report suggested that genetic screening of known candidate genes (MYOC, OPTN, and TBK1) in a single large South Indian family with POAG did not detect the genetic risk factors underlying the disease's pathogenesis. ${ }^{[21]}$ Therefore, this study aims to perform whole-exome sequencing (WES) to identify the potential genetic risk factors associated with the positive POAG family history of the five-generation south Indian family.

\section{Methods}

\section{POAG study subjects}

The study was approved by the Institutional Review Board at the Aravind Eye Care System, Madurai, Tamil Nadu, India (IRB2011008BAS). This research adhered to the tenets of the Declaration of Helsinki. All the study subjects were recruited and clinically evaluated as previously described. ${ }^{[21]}$ Briefly, an ophthalmic examination was conducted for 240 subjects during a field trip to Kayalpatanam; for this current study, 84 members were recruited from a single large South Indian family of five generations with a positive history of POAG [Supplementary Table 1].

Whole-exome sequencing

For WES, $5 \mathrm{~mL}$ of peripheral blood was collected from each study subject. The genomic DNA was extracted using a salting-out precipitation method ${ }^{[22]}$ and the concentration of the DNA samples was quantified using Qubit fluorometer. Samples were subjected to WES using the Agilent's SureSelect Human All Exon V6 kit. The DNA libraries have been sequenced to mean $>150 \times$ coverage on an Illumina HiSeq 4000 platform.

\section{Data analysis}

We developed an automated pipeline [Supplementary Fig. 1] to identify pathogenic variants from WES data using UNIX script (https://github.com/bharani-lab/WES-pipelines/tree/ master/Script). Raw reads (FASTQ file) were processed to remove the adapter and low-quality sequences using Cutadapt. Then the reads were further aligned against the human genome build GRCh37 using BWA-mem version 0.7.12. GATK version 4.1.0. for the identification of single-nucleotide variant (SNV) and small Insertion and Deletion (InDel) and it was further annotated using ANNOVAR. ${ }^{[23]}$ We first filtered rare and low-frequency variants keeping minor allele frequency (MAF) less than or equal to $0.5 \%$ in 1000 genome, ESP, ExAC, and gnomAD. Next, all the protein-coding variants that are either introducing or removing stop codon, altering transcripts (frameshift InDels), altering a canonical splice acceptor or splice donor site, and introducing an amino acid change (non-synonymous/missense variant) selected. The non-synonymous variants were further filtered as deleterious variants with a two-step process; firstly, variants were selected with the conservation score $>2.5$ (GERP score) and CADD score greater than 10; secondly, the variants should be predicted to be deleterious with at least three prediction tools among the five (Polyphen2, SIFT, Mutation Taster, FATHMM

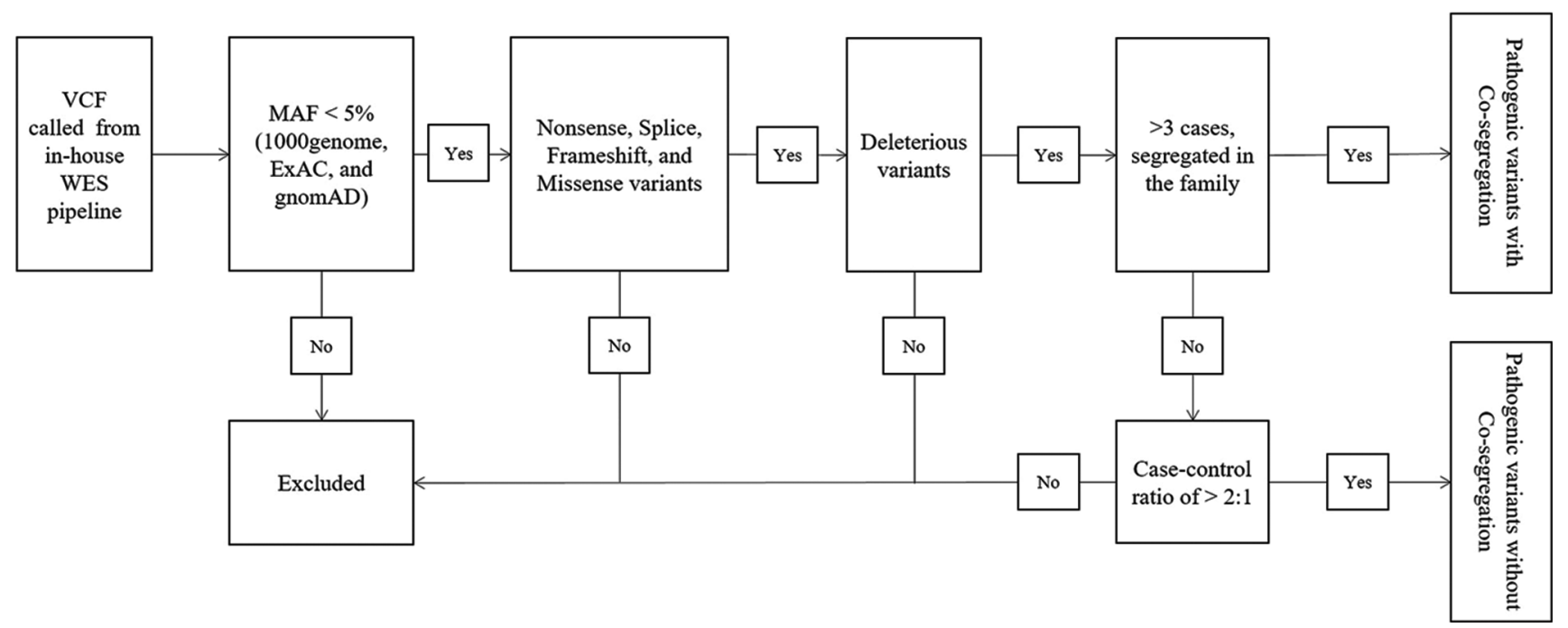

Figure 1: Workflow for variant prioritization

\begin{tabular}{|c|c|c|c|c|c|c|c|}
\hline $\begin{array}{l}\text { Chromosome } \\
\text { position }\end{array}$ & $\begin{array}{c}\text { Accession } \\
\text { number }\end{array}$ & $\begin{array}{l}\text { Nucleotide } \\
\text { changes }\end{array}$ & Gene name & $\begin{array}{l}\text { Amino acid } \\
\text { change }\end{array}$ & dbSNP & Varlect & Number of cases (sample ID) \\
\hline $14: 21816432$ & NM_020366.3 & c.G3719A & RPGRIP1 & p.G1240E & rs34725281 & $8.35^{\dagger}$ & 6 (III-3; III-2; II-2; III-32; IV-26; IV-27) \\
\hline $14: 21550588$ & NM_001278529.2 & c.A1295G & ARHGEF40* & p.Q432R & . & 1.59 & 6 (III-3; III-2; II-2; III-32; IV-26; IV-27) \\
\hline $14: 20666340$ & NM_001005503.1 & c. $847 \mathrm{delC}$ & OR11G2 & p.H282fs & rs528205284 & 0.99 & 6 (III-3; III-2; II-2; III-32; IV-26; IV-27) \\
\hline $14: 20482998$ & NM_001004712.1 & c.A355G & OR4K14 & p.M119V & rs7157076 & $0.95^{*}$ & 6 (III-3; III-2; II-2; III-32; IV-26; IV-27) \\
\hline $14: 21502110$ & NM_001012264.4 & c.C338T & RNASE13 & p.S113F & rs114504351 & $0.71^{*}$ & 6 (III-3; III-2; II-2; III-32; IV-26; IV-27) \\
\hline 14:19378312 & NM_001013354.1 & c.T719G & OR11H12 & p.V240G & rs61969158 & 0.22 & 6 (III-3; III-2; II-2; III-32; IV-26; IV-27) \\
\hline
\end{tabular}

Varlect score with symbol †represents the direct association with glaucoma phenotypes and ${ }^{\star}$ represent the indirect association. ${ }^{*}$ Represent the Novel variant 


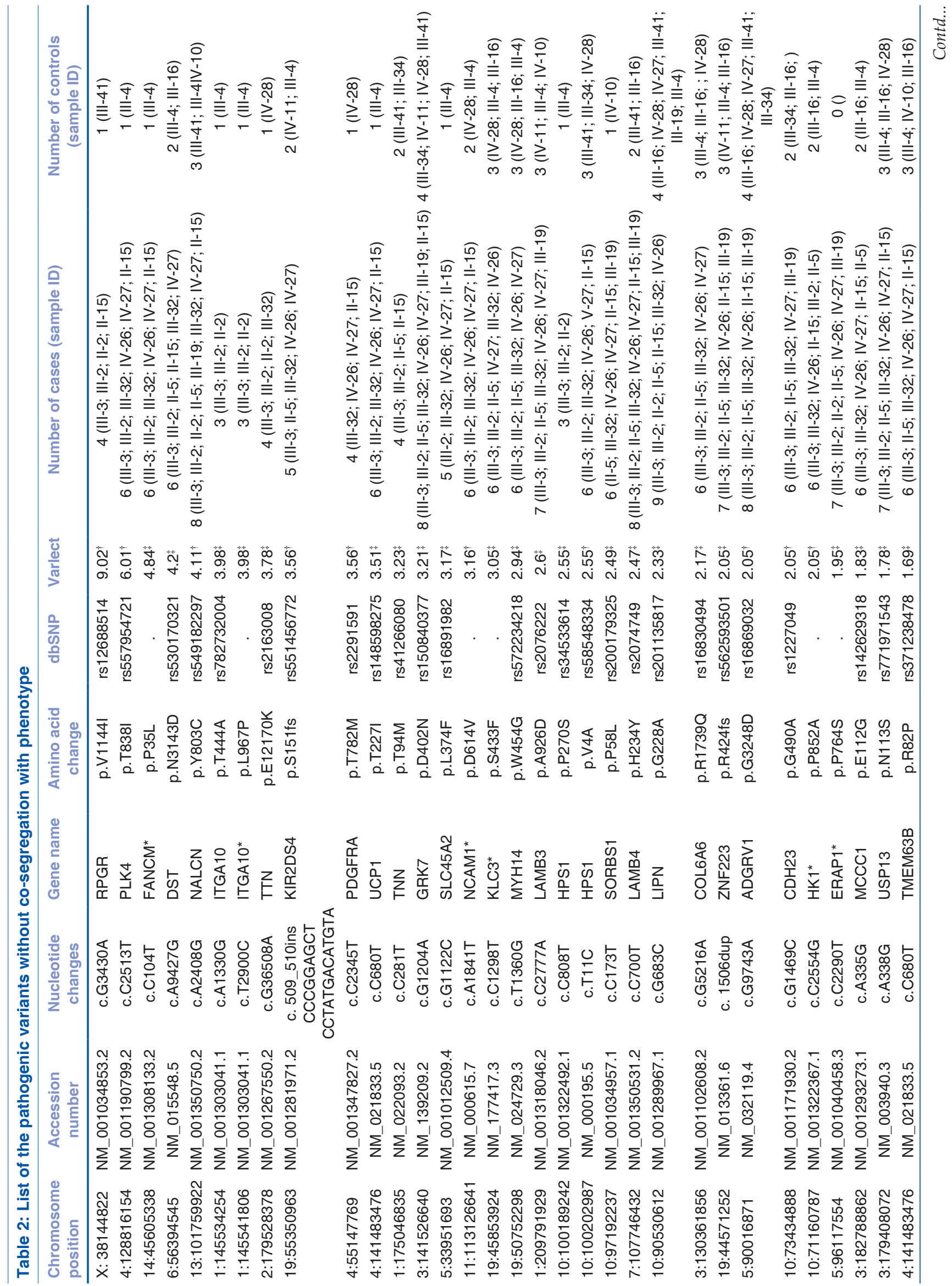




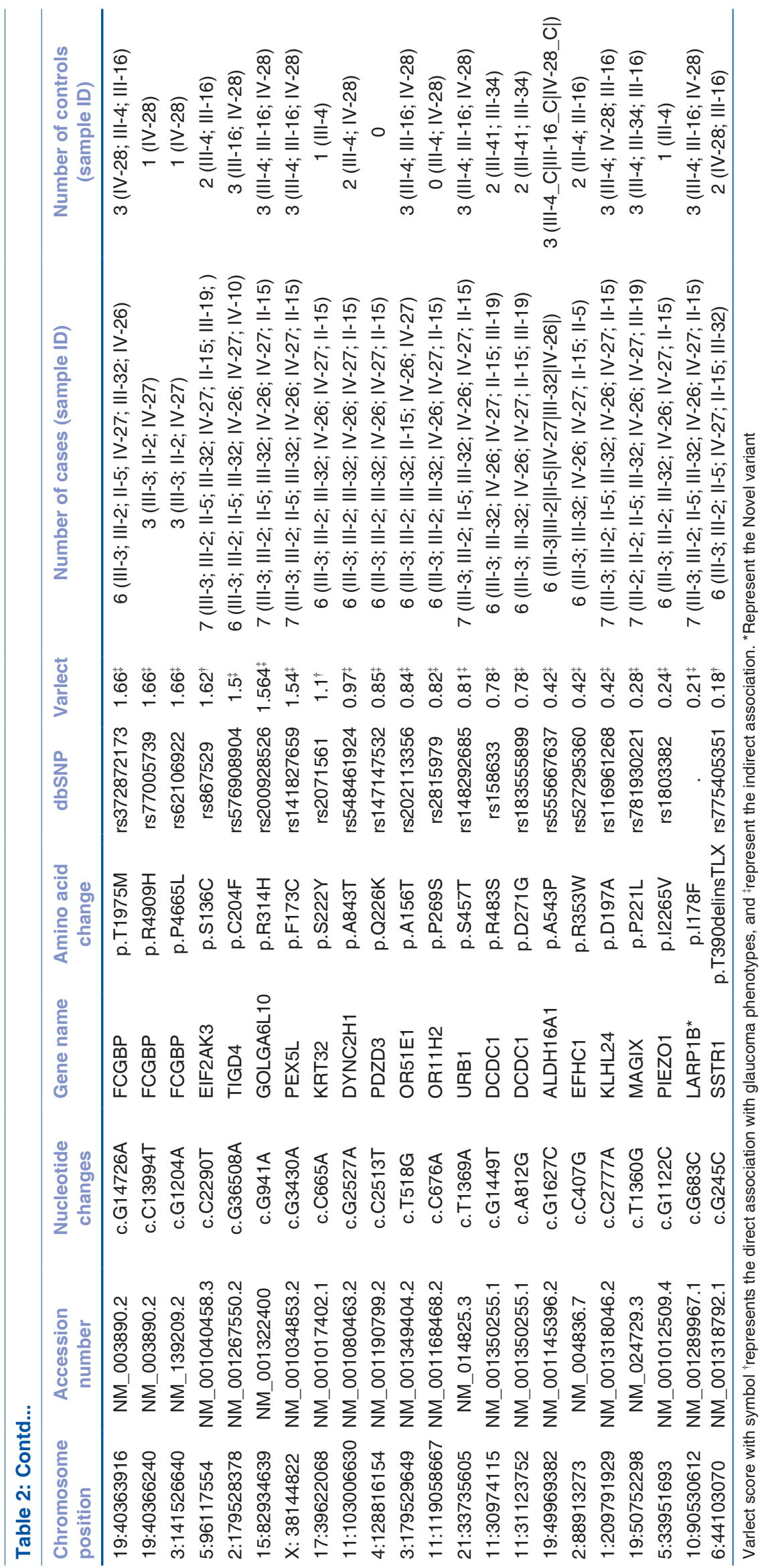




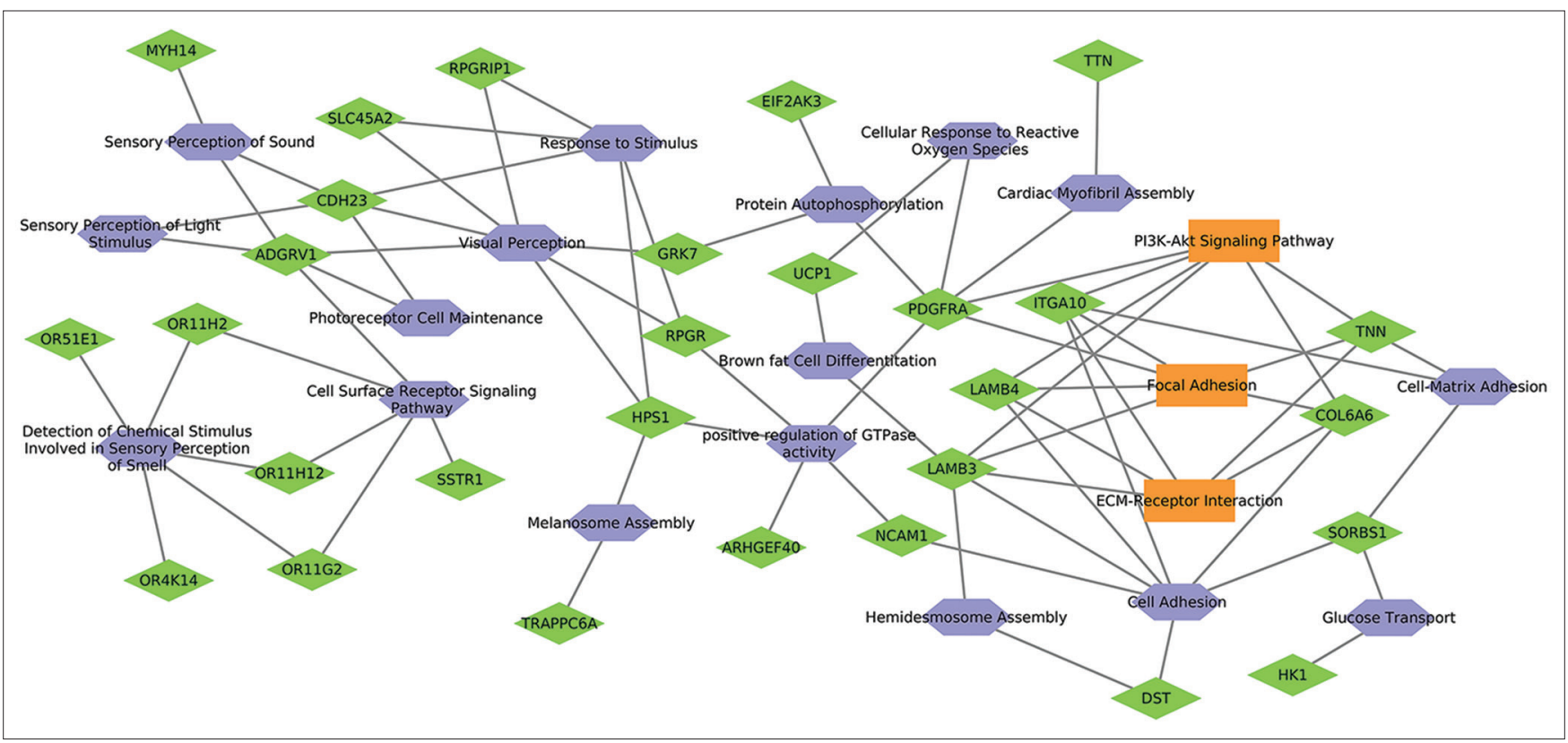

Figure 2: Functional network enriched with pathways and gene ontology (GO) on genes identified with pathogenic variants. Hexagon represents the gene, rectangle represents pathways, and diamond represents GO

and LRT). Also, we checked all the variants manually with the help of IGV viewers to avoid mapping errors. All predicted deleterious variants were further filtered based on their presence in at least more than three affected individuals in the pedigree. Finally, the variants were sorted out based on their segregation among their affected individuals. We used VarElect software ${ }^{[24]}$ to sort the genes based on their direct or indirect association with glaucoma.

We performed pathway and gene ontology analysis using DAVID for all the genes identified with pathogenic variants. A gene network was created using Cytoscape with the enriched pathways and biological processes.

\section{Sanger sequencing for the validation of novel variant}

For segregation analysis, the novel variant of the ARHGEF40 gene was PCR amplified using the following gene-specific primers (FW-5'-CTGAGCTGACGCCTGAAC TT-3'); (RV-5'-GCCGTGGGTACTGAGAAAG- 3') and the fragments were bi-directional sequenced using (3130 Genetic Analyser; Applied Biosystems). Further, the results were compared with the reference sequence of ARHGEF40 gene using NCBI-BLAST program and the chromatogram was analyzed using Chromas lite (2.1) software.

\section{Results}

\section{Clinical evaluation of patients}

A total of 84 family members were recruited from a single large south Indian family of five generations with a positive family history of POAG after a comprehensive ophthalmic screening of 240 family members in Kayalpatanam [as shown in Supplementary Fig. 2]. Clinical assessment and complex pedigree analysis revealed that 19 of the 84 samples had been diagnosed with POAG. The clinical features of all 19 POAG-affected individuals were discussed in detail ${ }^{[21]}$ [Supplementary Table 1].
Exome sequencing and variant filtering

Samples for the WES were selected solely based on the POAG inheritance pattern observed in the pedigree of the South Indian family, which included nine POAG cases belonging to the generation (II-2,5,15, III-2,3,19,32, and IV-26,27) and seven unaffected controls (II-5, III-4, 16, 34, 41, and IV-11, 28) [Supplementary Fig. 2]. WES was carried out using Agilent SureSelect Human All Exon V6 kit and the DNA libraries have been sequenced to the Illumina HiSeq 4000 platform with an average coverage depth of $\sim 150 \times$. The raw data were processed and analyzed to identify the pathogenic variants [as indicated in the methods section of Fig. 1]. Approximately 60,000 variants (SNV and InDel) were identified in each patient's exome aligned to the human reference genome build GRCh37.

\section{Pathogenic variants}

Based on pathogenic variant prioritization and phenotype-based sorting, we identified six pathogenic variants (5 non-synonymous, one frameshift variant) as shown in Table 1. We found a heterozygous variant c.G3719A altering amino acid (p.G1240E) with a deleterious effect that might affect the Retinitis Pigmentosa GTPase regulator-interacting protein1 (RPGRIP1) gene, which showed a direct association with glaucoma disease (Varlect score of 8.35). Among the WES screening, the variant was segregated in the family with the phenotype [Supplementary Fig. 3]. Also, Fernández-Martínez et al. ${ }^{[25]}$ showed that mutations in the RPGRIP1 gene might cause or increase the susceptibility to various forms of glaucoma, including POAG. Followed by the RPGRIP1 gene, we found a novel variant c.A1295G (p.Q432R) in the ARHGEF40 gene, which is also segregated with the family's phenotype. However, further confirmation by Sanger sequencing in the other family members (8 POAG and two unaffected family members) confirmed the same variant in 14 POAG cases and two unaffected family members. The other pathogenic genes were OR11G2 (c. 847delC p.H282fs), OR4K14 (c.A355G p.M119V), RNASE13 (c.C338T p.S113F), and OR11H12 (c.T719G p.V240G). 
Interestingly, all the pathogenic variants were found in the genetic loci of chromosomal location between14q19 and 14q21.

In addition to these, in this study, we also identified 54 variants as likely pathogenic variants in 54 genes, of which 51 were missense and two were InDel variants, as shown in Table 2. From the top list based on the glaucoma phenotype, RPGR gene variants may affect its protein partner RPGRIP1 in the RPGR proteasome complex. ${ }^{[26]}$ Mutations in PLK4, encoding a master regulator of centriole biogenesis, cause microcephaly, growth failure, and retinopathy. ${ }^{[27]}$ Interestingly, six variants were identified as novel variants. The top variant (c.A1841T p.D614V) in the neural cell adhesion molecule 1 (NCAM1) gene was further confirmed in six POAG and two unaffected family members by Sanger sequencing. The NCAM1 has been reported to be altered in the optic nerve, which is associated with elevated intraocular pressure. ${ }^{[28]}$

\section{Functional network analysis}

A functional network has been developed for all the pathogenic variants identified in POAG-affected individuals to investigate the pathways and biological processes involved in glaucoma pathogenesis. Initially, the DAVID database was used to integrate all genes with KEGG pathways and Gene Ontology (GO) process. 57 genes were significantly enriched into three pathways and $17 \mathrm{GO}$ biological processes $(P<0.01)$. These pathways include Focal adhesion, ECM-receptor interaction, and PI3K-Akt signaling pathway. Further in the Gene-functional network [Fig. 2, NCAM1, LAMB4, and PDGFRA genes connected all three pathways to other GO processes. Of these genes, NCAM1 was connected to the top gene list RPGRIP1 and ARHGEF40 with pathogenic variants through RPGR protein interaction and GO processes of positive regulation of GTPase activity and visual perception.

\section{Discussion}

Studies of larger pedigree in POAG diagnosed families led to discovering mutation in MYOC, OPTN, and TBK1 genes. Ophthalmic examination of a single family in South India with 84 family members over five generations with Egyptian heritage had a positive POAG family history revealed no mutation for the primary candidate genes (MYOC, OPTN, and TBK1) associated with POAG. ${ }^{[21]}$

Hence WES of 16 samples including (nine POAG and seven unaffected controls) of the 84 family members displayed a consistent co-segregation of six pathogenic genes ARHGEF40, RPGRIP1, OR4K14, RNASE13, OR11H12, and OR11G2 in six POAG samples. No pathogenic variants have been identified in three of the 9 POAGs and the remaining seven unaffected individuals. Furthermore, candidate genes for the three individuals diagnosed with POAG can be identified through either deep intronic or whole-genome sequencing. All the pathogenic variants identified from WES were further prioritized based on the glaucoma phenotype using a VarElect phenotype sorting tool. Among the six co-segregating pathogenic variants, only two (ARHGEF40 and RPGRIP1) showed association with glaucoma. The exciting fact is all six pathogenic variants were present in chromosome $14 q$, which had previously been reported to have potential POAG loci. ${ }^{[10,29,30]}$ However, we did not identify rare segregating variants in other genetic loci associated with POAG (CDKN2B-AS, CAV1/CAV2, TMCO1 AFAP1, TXNRD2,
FOXC1/GMDS, ATXN2, FNDC3B, GAS, PMM2, TGFBR3, and SIX1/SIX6).

The pathogenic variant in retinitis pigmentosa GTPase regulator-interacting protein 1 (RPGRIP1) gene is observed with the highest phenotype score in six POAG cases, suggesting that it may have a prominent role in POAG disease pathogenicity. Fernández-Martínez et al. ${ }^{[25]}$ has shown that the heterozygous non-synonymous variants in C2 domain of RPGRIP1 gene might cause various forms of glaucoma, including POAG. Also, it has demonstrated that RPGRIP1 interaction with NPHP4 protein was shown to play a key role in glaucoma pathogenesis. ${ }^{[25]}$ In this study, four POAG cases were found to have a heterozygous pathogenic missense variant in the RPGR gene. In contrast to this study homozygous or compound heterozygous variants detected in RPGRIP1 are also associated with photoreceptor dystrophies. ${ }^{[31,32]}$

Interestingly, we observed a pathogenic variant in RPGR gene, which is existed in four POAG cases. RPGRIP1 and its interacting partner RPGR, have been shown to express in the human retina and outside of the retina ${ }^{[26,33-35]}$ may regulate cilia genesis, maintenance, and function mainly through signaling pathways. ${ }^{[36]}$ Luo et al. ${ }^{[37]}$ reported that the primary cilia of trabecular meshwork (TM) mediates intraocular pressure regulation through signaling pathway in the eye and further highlighted that the signaling pathway defect leads to Lowe syndrome developed congenital glaucoma at birth. RPGR and its protein partners play an important role in actin cytoskeleton remodeling of cilia through these signaling pathways by activating the small GTPase, RhoA. ${ }^{[38]}$

This research also identified a novel pathogenic variant in the ARGHEF40 gene and this variant was further confirmed in all the affected family members using Sanger sequencing [Supplementary Fig. 3]. Studies show that Rho guanine nucleotide exchange factors Gene Family protein (ARHGEF12) has been implicated as a risk factor of glaucoma by increasing intraocular pressure through RhoA/RhoA kinase pathway. ${ }^{[39]}$ Furthermore, the Rho/ROCK pathway's activation results in trabecular meshwork (TM) contraction, and the inhibition of this pathway would aggravate TM's relaxation with a consequent increase in outflow facility and, thereby, decrease intraocular pressure. ${ }^{[40]}$ In the present study, we speculate that the ARGHEF40 variant may affect the RhoA signaling through RPGRIP1 and its interacting partner RPGR in actin cytoskeleton remodeling TM cilia, which may subsequently increase the intraocular pressure.

The pathogenic variants detected in other genes have not been reported to be directly associated with POAG. Therefore, we constructed a network of genes using GO and pathway enrichment. We have shown three pathways Focal adhesion, ECM-receptor interaction, and PI3K-Akt signaling pathway associated with the pathogenesis of POAG. Furthermore, the highlighted genes ARHGEF40, RPGRIP1, and RPGR were enriched through visual perception and positive regulation of GTPase activity. Intriguingly, the genes NCAM1, HSP1, and PDGFRA, including ARHGEF40 and RPGR in the biological process of positive regulation of GTPase activity are prioritized as top pathogenic variants based on the phenotype score. A study has shown that NCAM participates in the optic nerve changes associated with elevated intraocular pressure. ${ }^{[28]}$ However, the future work of the study will demand larger 
case-controls, which will help to perform family-based association tests to strengthen our analysis.

\section{Conclusion}

Overall, this study presented a panel of pathogenic variants in multiple genes and their possible association with POAG pathogenesis in the five-generation South Indian family. Thus, our findings strongly suggested that WES of the five-generation South Indian family showed extreme genetic heterogeneity of POAG within the family and the identified pathogenic variants showed continuous co-segregation among POAG-affected individuals. Pathway analysis also displayed the association of the candidate genes involved in POAG pathogenesis. However, it requires a larger sample size to ensure the authentic association of these identified genetic variants in POAG-affected individuals.

\section{Ethics approval and consent to participate}

The study adhered to the tenets of the Declaration of Helsinki, and ethics committee approved on 28/01/2011 by the Institutional Review Board of the Aravind Eye Care System (IRB2011008BAS). All study participants read and signed informed consent after explaining the nature and possible significances of the study.

\section{Availability of data and material}

The pipeline lines used for analysis in this study and a detailed tutorial are openly available for the public at (https://github. com/bharani-lab/Wole-Exome-Analysis-Pipeline).

We have submitted the data that support the finding of this study to SRA project ID PRJNA555016. Data can be accessed upon request.

\section{Acknowledgments}

We thank all the patients and their families for their participation in this study. We are grateful to the Indian Council of Medical Research (ICMR) for their financial support. We are also thankful to Mr. Saravanan, Mrs. Kalarani, and Mrs. Muthu Selvi for helping us in recruiting the samples and maintaining the clinical data in our lab.

\section{Financial support and sponsorship}

This research was supported by the Indian Council of Medical Research, Government of India (2012-0383/F1).

\section{Conflicts of interest}

There are no conflicts of interest.

\section{References}

1. Resnikoff S, Pascolini D, Etya'ale D, Kocur I, Pararajasegaram R, Pokharel GP, et al. Global data on visual impairment in the year 2002. Bull World Health Organ 2004;82:844-51.

2. Quigley H, Broman AT. The number of people with glaucoma worldwide in 2010 and 2020. Br J Ophthalmol 2006;90:262-7.

3. Tham Y-C, Li X, Wong TY, Quigley HA, Aung T, Cheng C-Y. Global prevalence of glaucoma and projections of glaucoma burden through 2040 A systematic review and meta-analysis. Ophthalmology 2014;121:2081-90.

4. Thylefors B, Négrel AD, Pararajasegaram R, Dadzie KY. Global data on blindness. Bull World Health Organ 1995;73:115-21.

5. Vijaya L, George R, Baskaran M, Arvind H, Raju P, Ve Ramesh S, et al. Prevalence of primary open-angle glaucoma in an urban South
Indian population and comparison with a rural population The Chennai glaucoma study. Ophthalmology 2008;115:648-654.e1.

6. Fingert J. Primary open-angle glaucoma genes. Eye (Lond) 2011;25:587-95.

7. Rezaie T, Child A, Hitchings R, Brice G, Miller L, Coca-Prados M, et al. Adult-onset primary open-angle glaucoma caused by mutations in optineurin. Science 2002;295:1077-9.

8. Stone EM, Fingert JH, Alward WL, Nguyen TD, Polansky JR, Sunden SL, et al. Identification of a gene that causes primary open angle glaucoma. Science 1997;275:668-70.

9. Nakano M, Ikeda Y, Tokuda Y, Fuwa M, Omi N, Ueno M, et al. Common variants in CDKN2B-AS1 associated with optic-nerve vulnerability of glaucoma identified by Genome-Wide association studies in Japanese. PLoS One 2012;7:e33389.

10. Osman W, Low S-K, Takahashi A, Kubo M, Nakamura Y. A genome-wide association study in the Japanese population confirms 9p21 and 14q23 as susceptibility loci for primary open angle glaucoma. Hum Mol Genet 2012;21:2836-42.

11. Ramdas WD, Rizopoulos D, Wolfs RC, Hofman A, de Jong PT, Vingerling JR, et al. Defining glaucomatous optic neuropathy from a continuous measure of optic nerve damage-The optimal cut-off point for risk-factor analysis in population-based epidemiology. Ophthalmic Epidemiol 2011;18:211-6.

12. Wiggs J. The cell and molecular biology of complex forms of glaucoma: Updates on genetic, environmental, and epigenetic risk factors. Invest Ophthalmol Vis Sci 2012;53:2467-9.

13. Thorleifsson G, Walters GB, Hewitt AW, Masson G, Helgason A, DeWan A, et al. Common variants near CAV1 and CAV2 are associated with primary open-angle glaucoma. Nat Genet 2010;42:906-9.

14. van Koolwijk LM, Ramdas WD, Ikram MK, Jansonius NM, Pasutto F, Hysi PG, et al. Common genetic determinants of intraocular pressure and primary open-angle glaucoma. PLoS Genet 2012;8:e1002611.

15. Gharahkhani P, Burdon KP, Fogarty R, Sharma S, Hewitt AH, Martin S, et al. Common variants near ABCA1, AFAP1 and GMDS confer risk of primary open-angle glaucoma. Nat Genet 2014;46:1120-5.

16. Cooke Bailey JN, Loomis SJ, Kang JH, Allingham RR, Gharahkhani P, Khor CC, et al. Genome-wide association analysis identifies TXNRD2, ATXN2 and FOXC1 as susceptibility loci for primary open-angle glaucoma. Nat Genet 2016;48:189-94.

17. Hysi PG, Cheng C-Y, Springelkamp H, Macgregor S, Cooke Bailey JN, Wojciechowski R, et al. Genome-wide analysis of multiethnic cohorts identifies new loci influencing intraocular pressure and susceptibility to glaucoma. Nat Genet 2014;46:1126-30.

18. Liu Y, Vitart V, Burdon KP, Khor CC, Bykhovskaya Y, Mirshahi A, et al. Genome-wide association analyses identify multiple loci associated with central corneal thickness and keratoconus. Nat Genet 2013;45:155-63.

19. Chen Y, Lin Y, Vithana EN, Jia L, Zuo X, Wong TY, et al. Common variants near ABCA1 and in PMM2 are associated with primary open-angle glaucoma. Nat Genet 2014;46:1115-9.

20. Li Z, Allingham RR, Nakano M, Jia L, Chen Y, Ikeda Y, et al. A common variant near TGFBR3 is associated with primary open angle glaucoma. Hum Mol Genet 2015;24:3880-92.

21. Abdul Kader M, Namburi P, Ramugade S, Ramakrishnan SR, Krishnadas S, Roos BR, et al. Clinical and genetic characterization of a large primary open angle glaucoma pedigree. Ophthalmic Genet 2016;38:222-225.

22. Miller SA, Dykes DD, Polesky HF. A simple salting out procedure for extracting DNA from human nucleated cells. Nucleic Acids Res 1988;16:1215.

23. Wang J, Liu X, Zhong Y. Rho/Rho-associated kinase pathway in 
glaucoma (Review). Int J Oncol 2013;43:1357-67.

24. Stelzer G, Plaschkes I, Oz-Levi D, Alkelai A, Olender T, Zimmerman $\mathrm{S}$, et al. VarElect: The phenotype-based variation prioritizer of the GeneCards suite. BMC Genomics 2016;17:444.

25. Fernández-Martínez L, Letteboer S, Mardin CY, Weisschuh N, Gramer E, Weber BHf, et al. Evidence for RPGRIP1 gene as risk factor for primary open angle glaucoma. Eur J Hum Genet 2011;19:445-51.

26. Roepman R, Bernoud-Hubac N, Schick DE, Maugeri A, Berger W, Ropers H-H, et al. The retinitis pigmentosa GTPase regulator (RPGR) interacts with novel transport-like proteins in the outer segments of rod photoreceptors. Hum Mol Genet 2000;9:2095-105.

27. Martin C-A, Ahmad I, Klingseisen A, Hussain MS, Bicknell LS, Leitch A, et al. Mutations in PLK4, encoding a master regulator of centriole biogenesis, cause microcephaly, growth failure and retinopathy. Nat Genet 2014;46:1283-92.

28. Ricard CS, Pena JD, Hernandez MR. Differential expression of neural cell adhesion molecule isoforms in normal and glaucomatous human optic nerve heads. Brain Res Mol Brain Res 1999;74:69-82.

29. Fan BJ, Wang DY, Pasquale LR, Haines JL, Wiggs JL. Genetic variants associated with optic nerve vertical cup-to-disc ratio are risk factors for primary open angle glaucoma in a US Caucasian population. Invest Ophthalmol Vis Sci 2011;52:1788-92.

30. Wiggs JL, Allingham RR, Hossain A, Kern J, Auguste J, DelBono EA, et al. Genome-wide scan for adult onset primary open angle glaucoma. Hum Mol Genet 2000;9:1109-17.

31. Booij JC, Florijn RJ, ten Brink JB, Loves W, Meire F, van Schooneveld $\mathrm{MJ}$, et al. Identification of mutations in the AIPL1, CRB1, GUCY2D, RPE65, and RPGRIP1 genes in patients with juvenile retinitis pigmentosa. J Med Genet 2005;42:e67.
32. Dryja TP, Adams SM, Grimsby JL, McGee TL, Hong DH, Li T, et al. Null RPGRIP1 alleles in patients with Leber congenital amaurosis. Am J Hum Genet 2001;68:1295-8.

33. Castagnet P, Mavlyutov T, Cai Y, Zhong F, Ferreira P. RPGRIP1s with distinct neuronal localization and biochemical properties associate selectively with RanBP2 in amacrine neurons. Hum Mol Genet 2003;12:1847-63.

34. Ferreira P. Identification of novel murine- and human-specific RPGRIP1 splice variants with distinct expression profiles and subcellular localization. Invest Ophthalmol Vis Sci 2005;46:1882-90.

35. Mavlyutov TA, Zhao H, Ferreira PA. Species-specific subcellular localization of RPGR and RPGRIP isoforms: Implications for the phenotypic variability of congenital retinopathies among species. Hum Mol Genet 2002;11:1899-907.

36. Patnaik SR, Kotapati Raghupathy R, Zhang X, Mansfield D, Shu X. The role of RPGR and its interacting proteins in ciliopathies. J Ophthalmol 2015;2015:414781.

37. Luo N, Conwell MD, Chen X, Insinna CI, Westlake CJ, Cantor LB, et al. Primary cilia signaling mediates intraocular pressure sensation. Proc Natl Acad Sci U S A 2014;111:12871-6.

38. Gakovic M, Shu X, Kasioulis I, Carpanini S, Moraga I, Wright A. The role of RPGR in cilia formation and actin stability. Hum Mol Genet 2011;20:4840-50.

39. Abiko H, Fujiwara S, Ohashi K, Hiatari R, Mashiko T, Sakamoto N, et al. Rho-guanine nucleotide exchange factors involved in cyclic stretch-induced reorientation of vascular endothelial cells. J Cell Sci 2015;128:1683-95.

40. Wang K, Li M, Hakonarson H. ANNOVAR: Functional annotation of genetic variants from high-throughput sequencing data. Nucleic Acids Res 2010;38:e164. 


\begin{tabular}{|c|c|c|c|c|c|c|c|c|c|c|c|}
\hline \multicolumn{12}{|c|}{ Supplementary Table 1: Clinical data available for POAG study subject } \\
\hline \multirow[t]{2}{*}{$\begin{array}{l}\text { Pedigree } \\
\text { symbol }\end{array}$} & \multirow{2}{*}{$\begin{array}{c}\text { Age at } \\
\text { diagnosis } \\
\text { (years) }\end{array}$} & \multirow[t]{2}{*}{$\begin{array}{l}\text { Max IOP } \\
(\mathrm{mm} \mathrm{Hg})\end{array}$} & \multirow[t]{2}{*}{$\begin{array}{c}\text { CCT } \\
\text { (microns) }\end{array}$} & \multicolumn{2}{|c|}{$\begin{array}{l}\text { Cup-To-disc ratio } \\
\text { at first exam }\end{array}$} & \multicolumn{4}{|c|}{$\begin{array}{l}\text { Humphrey visual field analyzer Data } \\
\text { (SITA 24-2/10-2*) }\end{array}$} & \multirow[t]{2}{*}{$\begin{array}{l}\text { Glaucoma } \\
\text { surgeries }\end{array}$} & \multirow[t]{2}{*}{ Comorbidity } \\
\hline & & & & OD & os & $\begin{array}{l}\text { MD OD } \\
\text { (dB) }\end{array}$ & $\begin{array}{l}\text { PSD OD } \\
\text { (dB) }\end{array}$ & $\begin{array}{c}\text { MD OS } \\
\text { (dB) }\end{array}$ & $\begin{array}{l}\text { PSD OS } \\
\text { (dB) }\end{array}$ & & \\
\hline III-2 & 23 & 24 & 522 & 0.7 & 0.4 & -2.89 & 8.07 & -0.26 & 1.1 & None & None \\
\hline III-3 & 34 & 26 & 527 & 0.7 & 0.8 & -8.36 & 8.36 & -10.37 & 10.01 & None & None \\
\hline II-2 & 65 & 15 & 506.5 & 0.8 & 0.8 & -6.05 & 2.42 & -9.05 & 4.93 & None & None \\
\hline $\mathrm{III}-20$ & 45 & 21 & 565.5 & 0.7 & 0.6 & -7.32 & 4.07 & -9.64 & 5.71 & None & None \\
\hline III-21 & 40 & 14 & 519 & 0.6 & 0.6 & NA & NA & NA & NA & None & None \\
\hline II-5 & 56 & 17 & NA & 0.9 & NA & NA & NA & NA & NA & Trabeculectomy & CRVO \\
\hline III-19 & 51 & 24 & 552 & 0.5 & 0.6 & -8.8 & 6.61 & -10.09 & 8.37 & None & None \\
\hline III-32 & NA & 26 & 519 & 0.9 & 0.9 & NA & NA & NA & NA & None & None \\
\hline IV-26 & NA & 36 & 528.5 & 0.9 & 0.9 & NA & NA & NA & NA & Trabeculectomy & CRVO \\
\hline IV-27 & NA & 18 & 617 & 0.8 & 0.8 & NA & NA & NA & NA & None & None \\
\hline III-38 & 68 & 22 & 535 & 0.6 & 0.6 & -3.63 & 1.63 & -4.42 & 2.24 & None & None \\
\hline III-26 & 66 & 18 & 486 & 0.8 & 0.8 & -14.24 & 8.12 & -12.56 & 9.56 & None & CRVO \\
\hline III-25 & 48 & 20 & 470 & 0.9 & NA & -26.62 & 9.97 & NA & NA & Trabeculectomy & CRVO \\
\hline II-15 & 53 & 34 & NA & NA & 0.9 & NA & NA & -23.57 & 8.25 & Trabeculectomy & CRVO \\
\hline Mean & 50 & 22.5 & 529 & \multicolumn{2}{|c|}{0.74} & -6.18 & 5.19 & -9.63 & 5.8 & NA & NA \\
\hline Std Dev & 14 & 6.5 & 37.8 & \multicolumn{2}{|c|}{0.14} & 2.46 & 2.90 & 7.19 & 3.31 & & \\
\hline
\end{tabular}

POAG=primary open-angle glaucoma, IOP=intraocular pressure, $C C T=$ central corneal thickness, SITA=Swedish interactive threshold algorithm, MD=mean deviation, $\mathrm{PSD}=$ pattern standard deviation, $\mathrm{NA}=$ not available, $\mathrm{CRVO}=$ central retinal vein occlusion. Nineteen family members were affected with $\mathrm{POAG}$. Five members were dead and not included in the table 


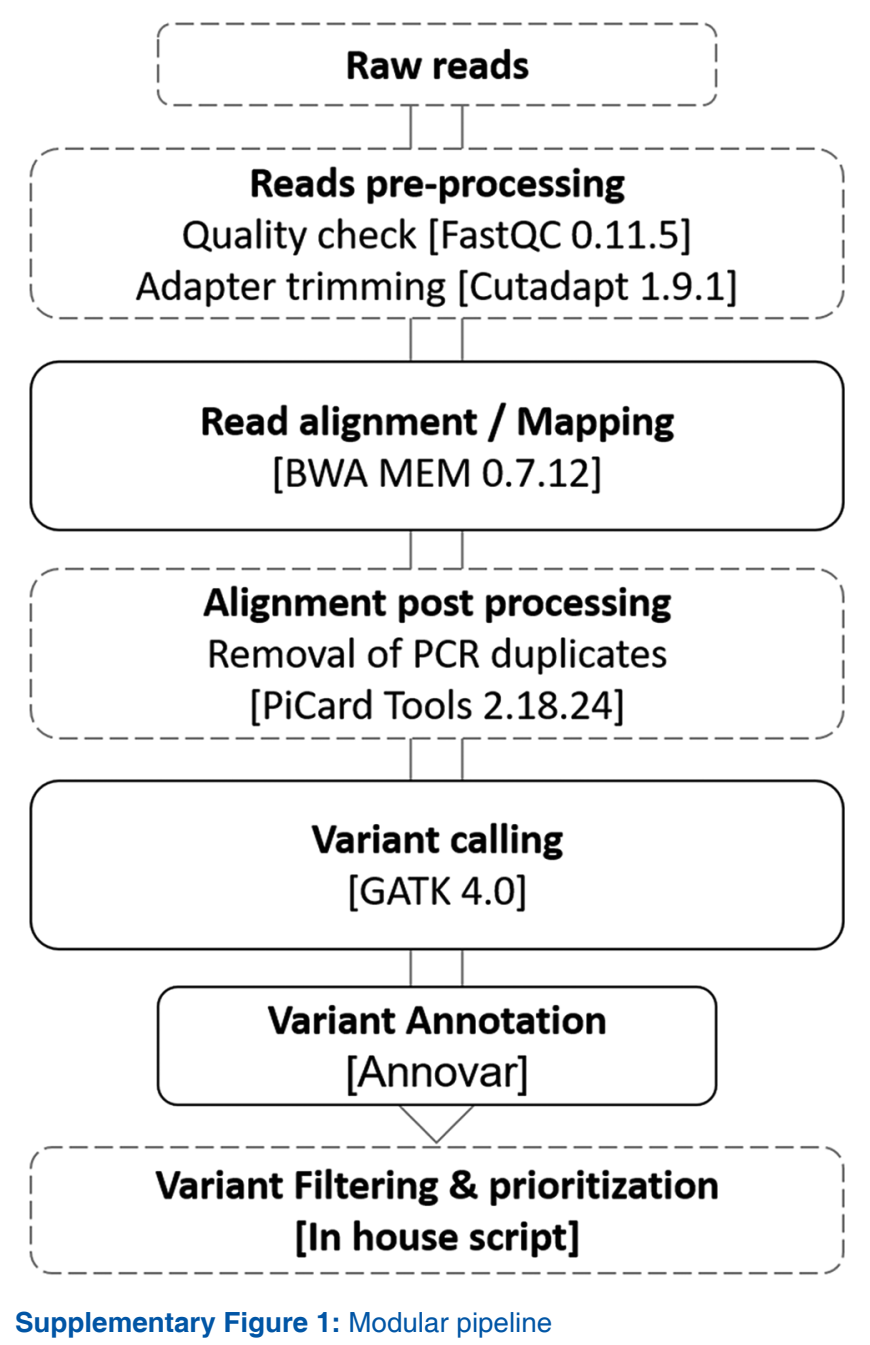




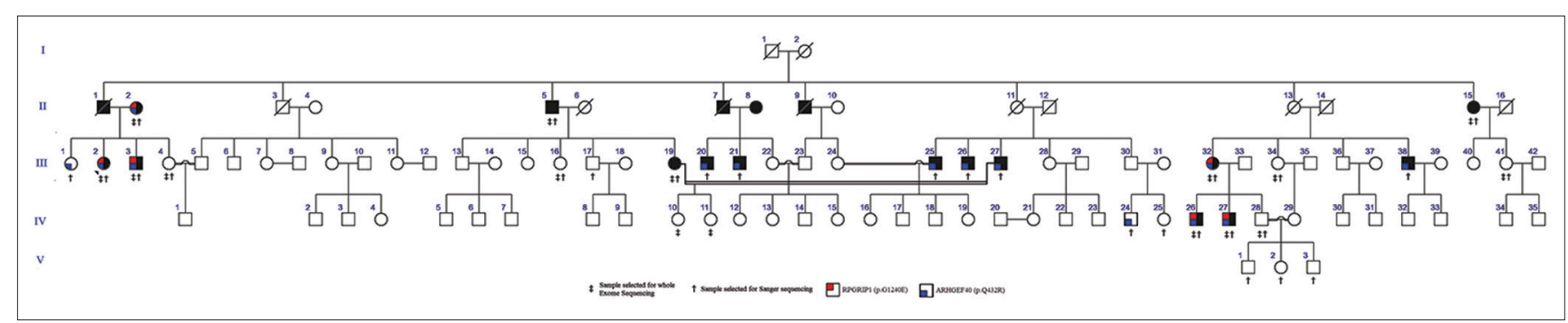

Supplementary Figure 2: Pedigree from south India Family. Family members diagnosed with primary open-angle glaucoma are shaded with black 


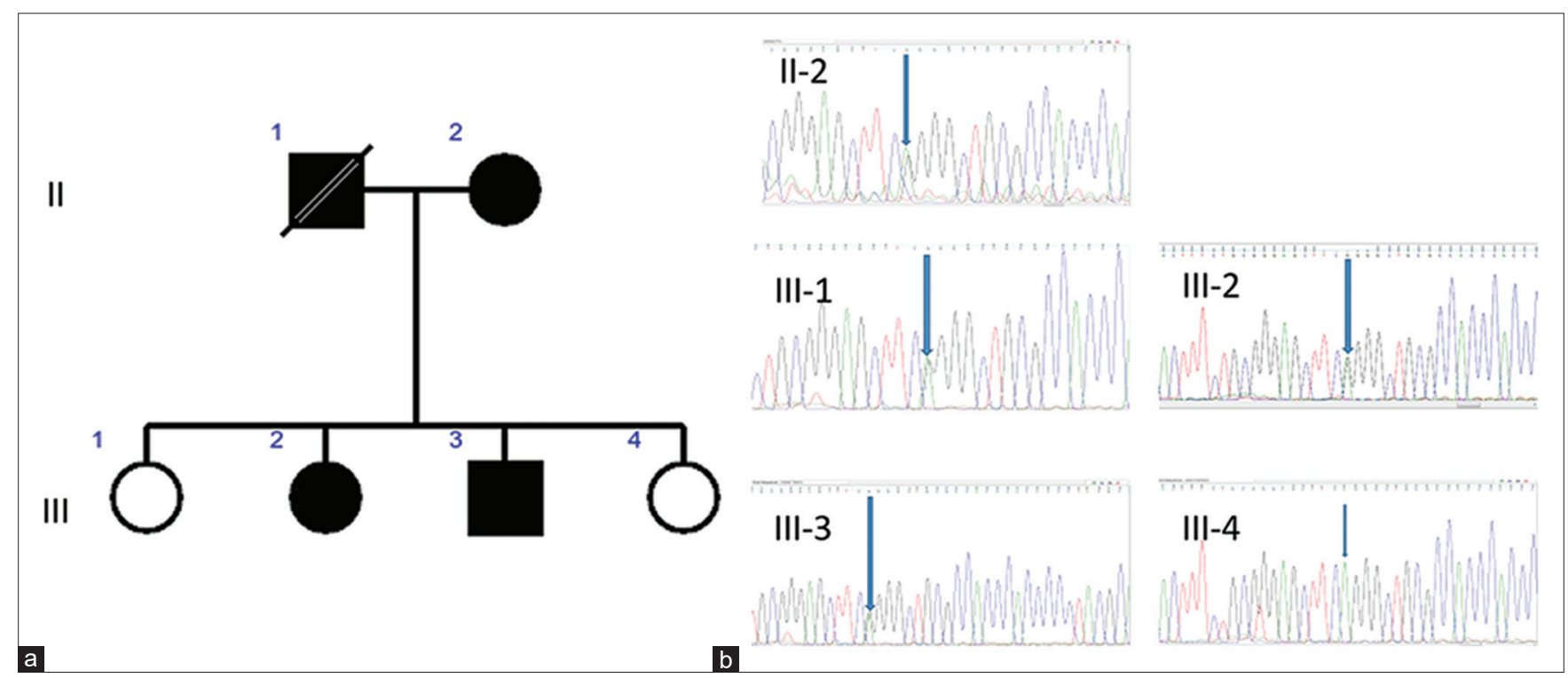

Supplementary Figure 3: Pedigree of selected family members from large south India family as shown in supplementary Figure 1 (a). Sanger sequencing results of novel variant c.A1295G in ARGEF40 gene (marked with down arrow). The variant is detected in the family members II-2, III-1, III-2, and III-3 (b) 